\title{
Análise das Complicações Tardias em Operações Anorretais: Experiência de um Serviço de Referência em Coloproctologia
}

\author{
Analysis of Late Complications of Anorectal Procedures: Experience of a \\ Referral Coloproctology Unit
}

\author{
JULIANA STRADIOTTO STECKERT ${ }^{1}$; MARIACRISTINA SARTOR ${ }^{2}$; ERONFÁBIO MIRANDA ${ }^{2}$; JULIANA \\ GONÇALVES ROCHA ${ }^{2}$; JULIANA FERREIRAMARTINS ${ }^{2}$; MARIACECÍLIAFERRAZDEARRUDASARTI \\ WOLLMANN $^{2}$; CRISTIANO DENONI FREITAS $^{1}$; ALVARO STECKERT FILHO $^{3}$; PAULO GUSTAVO KOTZE $^{4}$ \\ 1. Médicos residentes do Serviço de Coloproctologia do Hospital Universitário Cajuru- PUC-PR (SeCoHUC-PUCPR); \\ 2. Médicos do Staff do SeCoHUC-PUCPR; ${ }^{3}$ Acadêmico de medicina- Estagiário do SeCoHUC-PUCPR; ${ }^{4}$ Chefe do \\ $\mathrm{SeCoHUC}-\mathrm{PUCPR}$.
}

STECKERT JS; SARTOR MC; MIRANDA EF; ROCHA JG; MARTINS JF; WOLLMANN MCFAS; FREITAS CD; STECKERT FILHO A; KOTZE PG. Análise das Complicações Tardias em Operações Anorretais: Experiência de um Serviço de Referência em Coloproctologia. Rev bras Coloproct, 2010;30(3): 305-317.

RESUMO: Introdução: as operações anorretais correspondem a $80 \%$ do movimento do coloproctologista. 0 índice de complicações tardias após estas operações é indefinido, e varia de acordo com o tipo de operação e serviço onde estas são realizadas. Objetivo: estabelecer a taxa de complicações tardias decorrentes das operações anorretais e fatores de risco que pudessem estar associados a estas complicações. Método: estudo retrospectivo (série de casos) dos pacientes submetidos a operações anorretais entre janeiro de 2007 e julho de 2009. Variáveis estudadas: sexo, idade, operação, sistema de saúde, técnica de anestesia, complicações tardias, além da taxa de reoperações realizadas. Resultados: foram avaliados 430 pacientes $(234$ mulheres - 54,4\%), submetidos a 453 operações anorretais. A hemorroidectomia foi o mais freqüente procedimento realizado: 50,3\% das operações. Encontrou-se 102 complicações tardias pós-operatórias, representando 22,52\% dos casos. A fissura anal residual foi a complicação mais freqüente $(54 \% / n=55)$. Somente 38 pacientes necessitaram de reintervenção cirúrgica $(8,83 \%)$. Não houve diferença significativa em relação ao sexo, idade, sistema de saúde e ao tipo de operação realizada com as complicações encontradas. Conclusões: a taxa de complicações tardias foi de $22,52 \%$, com reintervenções cirúrgicas em $8,83 \%$ dos pacientes. Não houve fator de risco para complicações identificado nesta série de casos.

Descritores: Complicações pós-operatórias, hemorróidas, fístula retal, fissura anal, condiloma acuminado.

\section{INTRODUÇÃO}

As doenças anorretais são comuns na população geral. Estima-se que cerca de $5 \%$ da população adulta nos Estados Unidos é afetada por tais doenças ${ }^{1}$. Estas podem, em algumas situações, serem tratadas de forma conservadora. Todavia, o procedimento cirúrgico é necessário em vários casos selecionados. Dentre os principais tipos de tratamento cirúrgico des- critos, salientam-se as operações para tratamento das hemorróidas, fissuras, fístulas e abscessos perianais ${ }^{2}$.

As operações anorretais correspondem a cerca de $80 \%$ do total do movimento cirúrgico na especialidade de coloproctologia ${ }^{2}$. O porte dos procedimentos cirúrgicos anorretais é semelhante, independentemente da doença de base. O tempo operatório, materiais utilizados e cuidados de anestesia são equivalentes. 
A alta prevalência das doenças anorretais e o impacto econômico das várias formas de tratamento envolvidas têm estimulado o interesse pelo manejo ambulatorial dessas afecções ${ }^{3}$. Desta forma, nos últimos anos, a cirurgia proctológica ambulatorial tem recebido especial atenção devido à necessidade da diminuição dos custos hospitalares, da liberação de leitos para operações mais complexas, bem como diminuir as longas filas de espera para procedimentos cirúrgi$\cos 3,4,5$.

Apesar das operações anorretais serem procedimentos de tempo operatório relativamente curto e de pequeno porte, experiência e conhecimento da anatomia local são importantes para realizá-las. Mesmo nas mãos de especialistas hábeis e competentes, complicações cirúrgicas ocorrem com certa freqüência ${ }^{2,5}$. Sangramento retal, retenção urinária aguda, dor anal, fecaloma e infecção, são algumas das complicações precoces mais encontradas após estas operações ${ }^{6,7,8}$. Dentre as complicações tardias, as mais citadas na literatura são: incontinência fecal, nos variados graus de apresentação, fissuras e fístulas anais residuais, estenoses e recidivas das doenças ${ }^{6,7,8}$. Não se sabe exatamente a porcentagem de complicações tardias após operações anorretais. Este número é variável de acordo com o serviço, bem como com o tipo de operação realizada.

Há muito tempo as complicações após operações anorretais são descritas e estudadas. Em 1968, Granet descreveu seu descontentamento em reoperar muitos dos seus pacientes submetidos à hemorroidectomia, devido a fissuras anais residuais, estenoses e recidivas ${ }^{9}$. Refere ainda neste estudo, que seu colega, Fansler, reoperou $20 \%$ dos seus pacientes na época, devido a complicações tardias ${ }^{9}$.

Poucos são os trabalhos na literatura que avaliam as complicações tardias de várias operações anorretais associadas. Sobrado et al., na revisão de 503 operações proctológicas, evidenciaram 4,8\% de complicações tardias no geral. Entre as principais se destacam recidiva de fístula perianal $(0,8 \%)$, estenose anal $(0,4 \%)$, fissura residual $(0,4 \%)$ e incontinência fecal leve $(0,4 \% \text { dos casos })^{3}$. Saad-Hossne et al. avaliaram 437 procedimentos anorretais ambulatoriais, e encontraram 3\% de recidivas das entidades, $0,7 \%$ de incontinência fecal menor e 1\% de reoperações. Entretanto, estes autores não discriminaram qual tipo de intervenção cirúrgica apresentou maiores taxas de complicações dentre o grupo como um todo ${ }^{10}$. Prudente et al. também relataram uma série histórica de um serviço de coloproctologia, por 3 anos. Realizaram procedimentos anorretais em 455 pacientes, e encontraram 10,7\% de fissuras anais residuais e 3,5\% de incontinência para gases ${ }^{2}$.

As complicações precoces, geralmente, são atendidas no setor de emergência, ou até durante o internamento hospitalar. Por este motivo, estas podem ser melhor analisadas por meio de estudos prospectivos, pois a análise dos prontuários de ambulatórios apresentaria viés importante. Já as complicações tardias são analisadas nos retornos ambulatoriais dos pacientes, e são anotadas em prontuários, razão pela qual a análise por meio de estudo retrospectivo fica facilitada. Diante deste racional, há necessidade de estudos, para se analisar a incidência de complicações tardias dos procedimentos cirúrgicos proctológicos.

O objetivo principal deste estudo foi estabelecer a taxa de complicações tardias decorrentes das operações anorretais realizadas num serviço universitário. Os objetivos específicos foram: avaliar dados epidemiológicos associados aos pacientes submetidos a estas operações; taxas de complicações específicas de cada procedimento cirúrgico isolado; a taxa de reoperações dos pacientes portadores de complicações; e possíveis fatores de risco que pudessem estar relacionados a maiores taxas de complicações tardias nesta série de casos.

\section{MÉTODO}

O projeto de pesquisa do presente estudo foi aprovado pelo Comitê de Ética em Pesquisa da PUCPR (CEP - PUCPR), sob o parecer número 3395/09, que permitiu a análise retrospectiva das informações do banco de dados composto pelos prontuários destes pacientes.

Trata-se de um estudo descritivo, transversal. Este trabalho foi realizado no Serviço de Coloproctologia do Hospital Universitário Cajuru (SeCoHUC). Foram revisados retrospectivamente todos os prontuários médicos dos pacientes submetidos a operações anorretais, no período entre janeiro de 2007 e julho de 2009 (estudo retrospectivo de uma série de casos).

Foram incluídos pacientes dos sistemas público e privado de saúde, originários do ambulatório de coloproctologia do referido serviço e dos consultórios privados dos membros docentes. Os pacientes foram 
listados a partir de um livro ata, no qual constam todos os registros cirúrgicos do serviço.

Os critérios de exclusão para este estudo foram:

pacientes que não retornaram no ambulatório (ou consultórios) para seguimento, ou que o fizeram de maneira irregular, não comparecendo à consulta tardia dos 45 dias de pós-operatório.

pacientes menores de 18 anos.

prontuários com dados incompletos.

pacientes com doença de Crohn.

pacientes portadores do vírus da imunodeficiência humana (HIV).

Definiu-se como sucesso cirúrgico toda operação com tempo de seguimento maior que 45 dias, com alta ambulatorial, em pacientes assintomáticos. Definiu-se como complicação tardia, aquela encontrada no seguimento ambulatorial dos pacientes, após 45 dias (aproximadamente 7 semanas) de seguimento ou mais, considerando que o tempo de cicatrização destas cirurgias ocorrem entre 6 e 8 semanas.

A partir desta base de dados, os prontuários médicos ambulatoriais (e não das internações cirúrgicas) foram separados, e posteriormente analisados mediante preenchimento de protocolo específico. As variáveis estudadas foram: sexo, idade, operação realizada, comorbidades associadas, tempo de seguimento ambulatorial, técnica de anestesia utilizada, complicações tardias encontradas, além da taxa de reoperações realizadas nestes pacientes.

Todos os pacientes atendidos no ambulatório de coloproctologia e nos consultórios privados foram submetidos à anamnese geral e dirigida para a queixa principal, seguido de exame geral e proctológico (inspeção das regiões anal e perineal, além da realização de retossigmoidoscopia rígida). Quando necessário foram solicitados exames complementares, como enema opaco, colonoscopia, ultra-sonografia e endoscopia digestiva alta. Pelo caráter da instituição em que se realizou o estudo, universitária e de ensino, houve participação dos médicos residentes nas consultas e nos procedimentos cirúrgicos, sempre sob supervisão de um membro efetivo do serviço. Apenas as consultas realizadas nos consultórios privados, no pré e pós-operatório, tiveram participação exclusiva dos membros do corpo docente do serviço.

Após a indicação cirúrgica, os pacientes passavam por rotina de exames pré-operatórios e consulta pré-anestésica. Após a liberação por parte do anestesista, tinham seus procedimentos agendados. Os pacientes incluídos nesta análise não foram todos operados pelo mesmo cirurgião. Devido ao grande número de casos, equipes de cirurgia diferentes foram responsáveis por cada caso.

Durante o período citado, foram operados no referido serviço 1053 pacientes, incluindo-se operações anorretais e abdominais. Destes, 684 submeteram-se a procedimentos orificiais, somando $64,95 \%$ do movimento cirúrgico no período. Foram excluídos do trabalho 03 pacientes menores de 18 anos, 10 pacientes com doença de Crohn e 10 pacientes com portadores do vírus da imunodeficiência humana (HIV), 74 pacientes cujos prontuários não foram encontrados, 13 pacientes com prontuários incompletos, 60 pacientes que não possuíam seguimento algum no pós-operatório e 84 pacientes que não completaram 45 dias de pós-operatório, o que caracterizou seguimento incompleto. Totalizou-se, portanto, como amostra populacional deste estudo retrospectivo, 430 pacientes.

A análise estatística empregada para avaliação dos dados foi a análise descritiva com tabelas de freqüência. Foram calculadas medidas-resumo como média, mediana e desvio padrão, com auxílio do programa SPSS $^{\circledR}$ (SPSS Inc., Chicago IL, USA). Para se testar as hipóteses dos objetivos do estudo, foi utilizado o teste Chi-quadrado, com a avaliação de variáveis independentes. O nível de significância empregado (á) foi de $5 \%$.

\section{RESULTADOS}

Dos 430 pacientes analisados, 196 pacientes eram do sexo masculino e 234 do sexo feminino (figura 1). A média de idade encontrada foi de 44,3 anos. A idade mínima foi de 18 e máxima de 88 anos. A distribuição segundo faixas etárias é descrita na figura 2. Observou-se, nesta casuística, que $54,4 \%$ dos pacientes operados eram adultos jovens.

Foram realizadas, no total, 453 operações nos 430 pacientes. O número de procedimentos por paciente está descrito na tabela 1. No presente estudo, 9,3\% dos pacientes foram submetidos a mais de um procedimento cirúrgico (no caso, fistulotomias).

Houve distribuição semelhante entre o número de pacientes provenientes do sistema privado de saúde ( $\mathrm{n}=214 / 49,8 \%)$ se comparados aos do sistema público de saúde $(\mathrm{n}=216 / 50,2 \%)$. Estes dados estão detalhados na figura 3 . 
SEXO

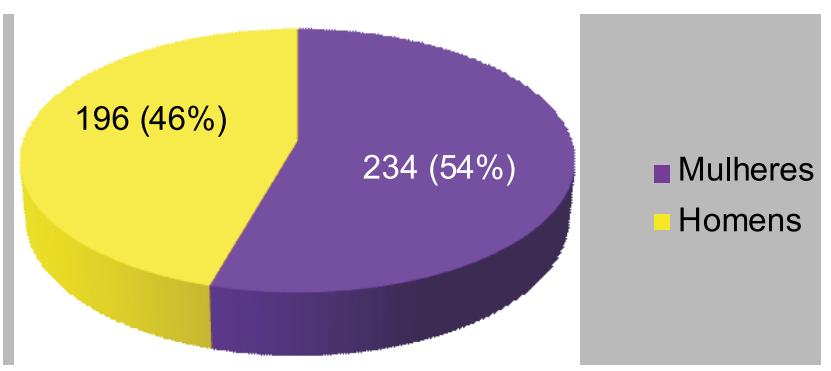

Figura 1 - Distribuição dos pacientes quanto ao sexo.

IDADE

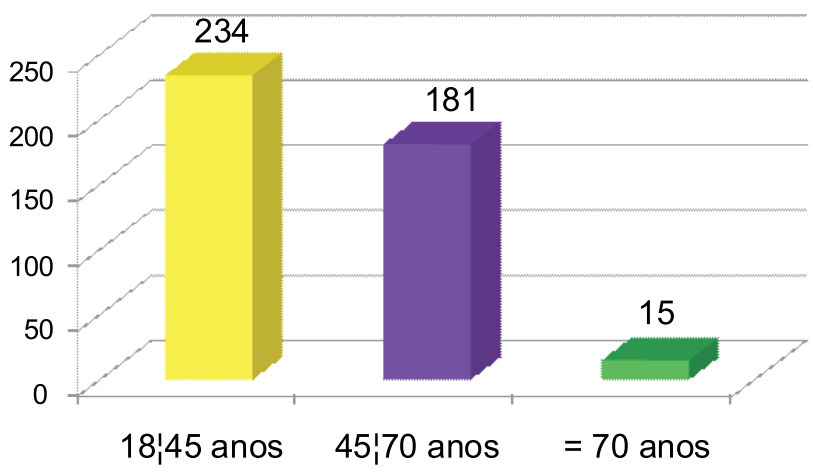

Figura 2 - Distribuição dos pacientes quanto à idade.

As operações realizadas estão descritas na tabela 2. Observou-se maior número de hemorroidectomias, pela alta incidência da doença hemorroidária, mais comum entidade com indicação cirúrgica nos procedimentos anorretais.
Origem dos Pacientes

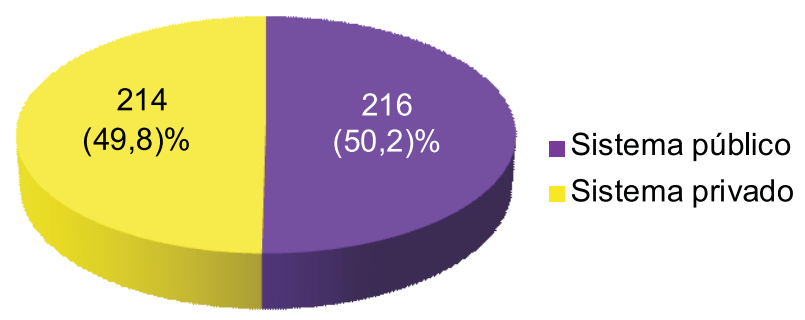

Figura 3 - Origem dos pacientes em relação ao sistema de saúde.

Tabela 1 - Distribuição do número de cirurgias por paciente.

\begin{tabular}{lrr}
\hline $\begin{array}{l}\text { Número de Operações } \\
\text { por paciente }\end{array}$ & n & \% \\
\hline $\mathbf{0 1}$ & 411 & 90,7 \\
$\mathbf{0 2}$ & 16 & 7,1 \\
$\mathbf{0 3}$ & 02 & 1,3 \\
$\mathbf{0 4}$ & 01 & 0,9 \\
Total & 453 & 100,0 \\
\hline
\end{tabular}

Quanto ao tipo de anestesia utilizada para estas operações, a maioria dos procedimentos realizouse em regime ambulatorial, com anestesia combinada por bloqueio perianal local e anestesia venosa com propofol $(n=374 / 82,56 \%)$. Apenas 79 pacientes

Tabela 2 - Operações anorretais realizadas na série de casos.

\begin{tabular}{lrc}
\hline Operações & $\mathbf{n}$ & \% \\
\hline Hemorroidectomia & 228 & 50,3 \\
Fistulotomias anais (em um e dois tempos) & 97 & 21,4 \\
Fissurectomias anais & 42 & 9,3 \\
Cauterização de condilomas & 24 & 5,3 \\
Drenagem de abscessos anais & 13 & 2,9 \\
Hemorroidectomia + Fissurectomia & 12 & 2,6 \\
Anopexia mecânica & 11 & 2,4 \\
Biópsias do canal anal & 10 & 2,2 \\
Esfincteroplastias anais & 06 & 1,3 \\
Anuplastias & 04 & 0,9 \\
Hemorroidectomia + Fistulotomia & 03 & 0,7 \\
Fissurectomia + Fistulotomia & 03 & 0,7 \\
Total & 453 & 100,0 \\
\hline
\end{tabular}


Análise das Complicações Tardias em Operações Anorretais: Experiência de um Serviço de Referência em Coloproctologia Juliana Stradiotto Steckert e Cols.
Vol. 30 $\mathbf{N}^{\mathbf{0}} 3$
$(17,44 \%)$ foram operados por meio de bloqueios locorregionais, com necessidade de internação hospitalar e pernoite.

O tempo médio de seguimento dos pacientes submetidos à operação anorretal foi de 164,7 dias, com tempo mínimo de 45 dias e máximo de 849 dias. Durante este período, identificaram-se 102 complicações tardias (22,52\% das operações incluídas neste estudo), as quais se encontram listadas na tabela 3. Dentre as encontradas, a principal foi fissura anal residual. A tabela 4 evidencia a hemorroidectomia como a principal causa da fissura anal residual, com significância estatística $(p<0,0001)$.

Apenas 38 dos 430 pacientes necessitaram de reoperações, no total dos pacientes incluídos nesta casuística $(8,83 \%$ dos casos) (tabela 5$)$. O tempo médio decorrido entre os dois procedimentos foi de 192,1 dias, variando entre 20 e 754 dias. Nas reoperações, as duas hemorroidectomias foram realizadas por recidiva da doença hemorroidária. Dos 55 pacientes portadores de fissuras anais residuais, somente $8(14,5 \%)$ necessitaram de correção cirúrgica (6 fissurectomias + 2 anuplastias). Os outros 47 casos obtiveram êxito com o tratamento clínico instituído.

Dentre os 21 casos de fístulas pós-operatórias, citados nas complicações, 16 foram operados, e 5 pacientes aguardam a correção cirúrgica na lista de espera do serviço. Os 3 pacientes com estenoses se beneficiaram com a realização da anuplastias, com resolução completa dos casos. Nas recidivas de condilomas anais, um paciente ainda aguarda por nova cauterização.

Entre os 14 pacientes com incontinência fecal leve no pós-operatório, definida por perda no controle de gases e secreção fecal, principalmente após as evacuações (escape), 5 apresentavam defeito esfincteriano, e tiveram indicação de esfincteroplastia anal. Destes, 3 já foram operados, 2 aguardam a realização de seus procedimentos cirúrgicos na lista de espera do serviço.

A análise estatística com o cruzamento de dados, comparando-se o sexo com as operações anorretais realizadas, demonstrou predominância do sexo feminino nas hemorroidectomias $(p=0,0001)$, e do sexo masculino nas fistulotomias anais $(p=0,033)$ e nas anopexias mecânicas $(p=0,035)$.

Comparando-se a distribuição das faixas etárias dentre os procedimentos cirúrgicos, percebe-se a formação de grupos heterogêneos nas hemorroidectomias
Tabela 3 - Complicações cirúrgicas tardias encontradas na série de casos.

\begin{tabular}{lrc}
\hline Complicações & n & \% \\
\hline Fissura anal residual & 55 & 54 \\
Incontinência anal leve & 14 & 13,7 \\
Fístula anal residual & 12 & 11,8 \\
Recidiva de fístula anal & 9 & 8,8 \\
Recidiva de condilomas & 7 & 6,8 \\
Estenose anal & 3 & 2,9 \\
Recidiva hemorroidária & 2 & 2 \\
Total & 102 & 100,0 \\
\hline
\end{tabular}

Tabela 4 - Operações anorretais associadas à fissura anal residual, principal complicação cirúrgica tardia encontrada, em 55 pacientes. Análise estatística pelo método de Chi-quadrado $(p=0,05)$. A hemorroidectomia foi associada a maiores taxas de fissura residual, com significância estatística.

\begin{tabular}{lcc}
\hline Operações & n & \% \\
\hline Hemorroidectomia & $35 *$ & $63,6^{*}$ \\
Fissuras anais & 9 & 16,4 \\
Fístulas anais & 4 & 7,3 \\
Hemorroidectomia+ Fissurectomia & 4 & 7,3 \\
Hemorroidectomia + Fistulotomia & 2 & 3,6 \\
Fissurectomia + Fistulotomia & 1 & 1,8 \\
Total & 55 & 100,0 \\
\hline
\end{tabular}

$* p<0,0001$

Tabela 5 - Reoperações realizadas para tratamento das complicações tardias encontradas.

\begin{tabular}{lrr}
\hline Reoperações realizadas & n & \% \\
\hline Fistulotomias anais & 16 & 42,1 \\
Fissurectomias anais & 6 & 15,8 \\
Cauterização de condilomas & 6 & 15,8 \\
Anuplastias & 5 & 13,1 \\
Esfincteroplastias anais & 3 & 7,9 \\
Hemorroidectomias & 2 & 5,3 \\
Total & 38 & 100,0 \\
\hline
\end{tabular}


Análise das Complicações Tardias em Operações Anorretais: Experiência de um Serviço de Referência em Coloproctologia Juliana Stradiotto Steckert e Cols.
Vol. 30 $\mathbf{N}^{\circ} 3$ $(p<0,0001)$, fissurectomias anais $(p<0,0001)$, fistulotomias anais $(p<0,0001)$ e cauterizações de condilomas anais $(p<0,0001)$. Não se consegue evidenciar qual a discrepância em cada grupo, devido ao número pequeno de pacientes no grupo acima de 70 anos.

Ao se analisar a presença de complicações cirúrgicas tardias encontradas com o sexo dos pacientes, não houve diferença estatística significativa $(p=0,53)$. Por este motivo, afirma-se que as complicações tardias tiveram prevalência semelhante entre homens e mulheres. Estes dados encontram-se detalhados na tabela 6 .

Da mesma forma, ao se analisar as faixas etárias estudadas com a presença de complicações cirúrgicas tardias, não houve diferença estatística significativa $(p=0,582)$. Afirma-se que as complicações ocorreram no presente estudo independentemente da faixa etária dos pacientes. Estes dados estão ilustrados na tabela 7 .

Da mesma for, analisando-se a procedência dos pacientes, relacionada à presença de complicações cirúrgicas, não houve diferença estatística significativa entre os pacientes do sistema público e os do sistema privado de saúde $(p=0,863)$ (tabela 8$)$.

Com relação às operações anorretais realizadas, associadas às suas complicações, não se evidenciou nenhuma diferença estatística entre as mesmas $(p=0,107)$. Portanto, nenhuma operação específica se destacou como responsável por maior número de complicações tardias. Estas se distribuíram de forma homogênea entre os variados tipos de operações. Estes dados encontram-se detalhados na tabela 9.

\section{DISCUSSÃO}

Apesar de suas elevadas prevalências, as doenças anorretais são freqüentemente consideradas como distúrbios de baixa complexidade de tratamento e reduzidos índices de morbimortalidade. Como conseqüência, poucos têm sido os estudos referentes à sua epidemiologia, elemento de grande importância para melhor compreensão de seus aspectos fisiopatológicos e de sua história natural. Há escassez de estudos que forneçam dados concretos em relação aos procedimentos cirúrgicos realizados e ao seguimento tardio destes pacientes ${ }^{11,12}$.

Sendo freqüentemente diagnosticadas e tratadas em âmbito de atenção primária à saúde, as entida-
Tabela 6 - Relação entre sexo e complicações tardias. Análise estatística pelo método de Chi-quadrado $(p=0,05)$.

\begin{tabular}{lrrrr}
\hline \multirow{2}{*}{ Sexo } & \multicolumn{3}{c}{ Complicações Tardias } \\
\cline { 2 - 5 } & \multicolumn{2}{c}{ Não } & \multicolumn{2}{c}{ Sim } \\
\cline { 2 - 5 } & $\mathbf{n}$ & $\%$ & $\mathbf{n}$ & $\%$ \\
\hline Feminino & 170 & 72,6 & 64 & 27,4 \\
Masculino & 158 & 80,6 & 38 & 19,4 \\
\hline
\end{tabular}

$p=0,53$

Tabela 7 - Relação entre faixa etária e complicações tardias encontradas. Análise estatística pelo método de Chi-quadrado $(p=0,05)$.

\begin{tabular}{lrrrr}
\hline Idade & \multicolumn{3}{c}{ Complicações Tardias } \\
\cline { 2 - 5 } & \multicolumn{2}{c}{ Não } & \multicolumn{2}{c}{ Sim } \\
\cline { 2 - 5 } & n & $\%$ & n & $\%$ \\
\hline $18 \$ \% 45$ anos & 177 & 75,6 & 57 & 24,4 \\
$45 \$ \% 70$ anos & 141 & 77,9 & 40 & 22,1 \\
e" 70 anos & 10 & 66,7 & 5 & 33,3 \\
\hline
\end{tabular}

$p=0,582$

Tabela 8 - Relação entre a procedência dos pacientes e complicações tardias. Análise estatística pelo método de Chi-quadrado $(p=0,05)$.

\begin{tabular}{lcccc}
\hline Procedência & \multicolumn{3}{c}{ Complicações Tardias } \\
\cline { 2 - 5 } & \multicolumn{2}{c}{ Não } & \multicolumn{2}{c}{ Sim } \\
\cline { 2 - 5 } & $\mathbf{n}$ & $\%$ & $\mathbf{n}$ & $\%$ \\
\hline Sistema público & 164 & 75,9 & 52 & 24,1 \\
Sistema privado & 164 & 76,6 & 50 & 23,4 \\
\hline
\end{tabular}

$p=0,863$

des anorretais passam por diagnósticos inadequados e freqüentemente se perpetuam de forma indiscriminada, o que gera uma indefinição de $\operatorname{conceitos}^{12}$. Tais dilemas prejudicam os pacientes, e uma enorme gama de sintomas provenientes da região anal é indistintamente atribuída a "hemorróidas" 11 . Diante do exposto, percebe-se a dificuldade de se desenvolver estudos epidemiológicos de escala maior, realizados por médicos especialistas em doenças anorretais ${ }^{12}$. 
Tabela 9 - Associação entre as operações realizadas e complicações tardias. Análise estatística pelo método de Chi-quadrado $(p=0,05)$.

\begin{tabular}{lrcrc}
\hline \multirow{2}{*}{ Operações realizadas } & \multicolumn{4}{c}{ Complicações Tardias } \\
\cline { 2 - 5 } & \multicolumn{3}{c}{ Não } & Sim \\
\cline { 2 - 5 } & $\mathbf{n}$ & 78,9 & $\mathbf{n}$ & \% \\
\hline Hemorroidectomia & 77 & 79,4 & 20 & 21,1 \\
Fistulotomia anal & 33 & 78,6 & 9 & 20,6 \\
Fissurectomia anal & 15 & 62,5 & 9 & 21,4 \\
Cauterização de condilomas anais & 10 & 90,9 & 1 & 37,5 \\
Anopexia mecânica & 10 & 100 & - & 9,1 \\
Biópsia do canal anal & 9 & 69,2 & 4 & - \\
Drenagem de abscessos & 6 & 50 & 6 & 30,8 \\
Hemorroidectomia + Fissurectomia & 5 & 83,3 & 1 & 50 \\
Esfincteroplastia & 3 & 75 & 1 & 16,7 \\
Anuplastia & 2 & 66,7 & 1 & 25 \\
Fissurectomia + Fistulotomia & 1 & 33,3 & 2 & 33,3 \\
Hemorroidectomia + Fistulotomia & 351 & 77,5 & 102 & 66,7 \\
Total & & & & 22,5 \\
\hline
\end{tabular}

$p=0,107$

Apesar das operações anorretais serem procedimentos de tempo operatório relativamente curto e de pequeno porte, experiência é importante para realizá-las adequadamente. Mesmo nas mãos de especialistas hábeis e competentes, complicações cirúrgicas ocorrem com certa frequiência ${ }^{2,5}$. Estas complicações podem ser simples ou graves, como por exemplo, a alteração de continência fecal, que pode interferir na qualidade de vida dos pacientes.

Poucos são os estudos na literatura que avaliam as complicações pós-operatórias de operações anorretais. Deste ponto surgiu a necessidade e o interesse de se confeccionar a presente casuística. Houve, da mesma forma, o propósito de se procurar por fatores de risco que pudessem influenciar nas taxas de complicações, para se aperfeiçoar a acurácia cirúrgica, resultando em maiores benefícios para os pacientes.

O número de pacientes submetidos a operações anorretais no referido serviço foi de 684 , num intervalo de aproximadamente 2,5 anos (64,5\% do movimento cirúrgico). Esta é a média encontrada na literatura, a qual identifica que em serviços de referência em Coloproctologia, $50 \%$ a $80 \%$ do movimento cirúrgico decorre de operações anorretais ${ }^{2}$. Prudente et al, também produzindo uma série histórica em um serviço de referência do nordeste do Brasil, computaram 72,5\% do movimento cirúrgico proveniente de operações anorretais ${ }^{2}$.

Na presente casuística, houve distribuição semelhante dos pacientes submetidos a operações anorretais quanto ao sexo. Foram 234 mulheres (54,5\%) e 196 homens (45,6\%). A amostra é semelhante aos achados literatura. Distribuição homogênea foi descrita por Nahas et al. ${ }^{5}$ (50,7\% do sexo feminino) ,Prudente et al. ${ }^{2}$ (54\% do sexo feminino) e Saad-Hossne et al. ${ }^{10}$ (56\% do sexo feminino), entre outros.

Ao se comparar a distribuição dos pacientes quanto ao sexo, separadamente entre os procedimentos cirúrgicos, observou-se uma prevalência maior do sexo feminino $(p=0,0001)$ no grupo das hemorroidectomias, e do sexo masculino no grupo das fistulotomias anais $(p=0,33)$ e da anopexia mecânica $(p=0,035)$. A literatura refere uma relação de 1,3 homens para cada mulher com doença hemorroidária ${ }^{6}$, ${ }^{12}$. A presença de um número maior de mulheres no grupo das hemorroidectomias pode ser justificada pelo maior número de diagnósticos feitos no sexo feminino. Há algumas justificativas para este fato. As mulheres se queixam para seus ginecologistas com mais facilidade e são encaminhadas mais precocemente para avaliação proctológica. Além disso, apresentam menor 
Análise das Complicações Tardias em Operações Anorretais

Experiência de um Serviço de Referência em Coloproctologia Juliana Stradiotto Steckert e Cols.
Vol. 30 pudor ao referir queixas anorretais, além da questão estética, atualmente muito valorizada pelas pacientes. A predominância do sexo masculino nas fistulotomias se deu, provavelmente, pela maior incidência de fístulas anais nos homens, fato amplamente descrito na literatura $2,8,12$.

A média de idade apresentada no grupo de pacientes deste trabalho foi de 44,3 anos. Este achado também é semelhante aos relatados nos demais estudos na literatura 2, 10,11,13,14,15,16. Optou-se por agrupar os indivíduos em 3 faixas etárias: entre 18 e 45 anos (54,4\% dos casos), entre 46 e 70 anos de idade $(42,1 \%)$ e maiores de 70 anos $(3,5 \%)$, conforme classificações de outros trabalhos referidos ${ }^{15,17}$. Não existe uma padronização ideal na divisão das idades. Alguns trabalhos apresentam somente as médias de idade ${ }^{3,5,14}$, outros intervalos de 10 anos $^{2,11}$, ou intervalos de 20 anos 18. Não houve diferença estatística entre as faixas etárias comparadas nesta casuística. Entretanto, pode existir um viés neste aspecto, pois o grupo representado pelos pacientes maiores de 70 anos foi muito pequeno.

Pode-se observar que a média de idade de 44,3 anos coincide com a média de idade da apresentação das doenças anorretais de maneira geral ${ }^{6,10,12}$, e que houve uma diminuição decrescente de acordo com a evolução das faixas etárias. Estes resultados coincidem com os encontrados por Pinho et al., ${ }^{12}$, que propuseram algumas justificativas para se explicar os resultados encontrados. Considerando-se que o acesso aos ambulatórios é o mesmo para a população geral, e que eventuais problemas de locomoção encontrados por parte dos pacientes mais idosos podem ser compensados por uma maior disponibilidade de tempo para consultas, parece claro que a frequiência dos atendimentos foi diretamente relacionada à necessidade de resolução de sintomas existentes, no estudo citado. Estes sintomas pareceram diminuir em incidência com o passar dos anos, de acordo com os autores ${ }^{12}$.

Avaliando-se a distribuição dos pacientes pelas faixas etárias, de acordo com os procedimentos realizados, houve diferença estatística significativa $(p<$ 0,0001) nos principais grupos: hemorroidectomias, fissurectomias, fistulotomias e cauterização de condilomas. Este viés pode ser justificado, da mesma forma, pelo fato do grupo de pacientes acima de 70 anos ter tido um número reduzido de pacientes.

No presente estudo, somaram-se 453 procedimentos cirúrgicos em 430 pacientes. Este achado não considera as reoperações, e sim, operações anorretais prévias. Do total, 9,3\% dos pacientes foram submetidos a mais de um procedimento cirúrgico, que variou entre 2 e 4 operações. Todo este grupo foi representado por pacientes que foram submetidos a drenagens de abscessos, seguidos de fistulotomias anais em um ou dois tempos. Foram 97 fistulotomias realizadas em 74 pacientes, portanto, uma média de 1,31 fistulotomias por paciente. $\mathrm{O}$ número encontrado no presente estudo é semelhante ao encontrado na literatura ${ }^{19,20}$. Davies et al., em um estudo com 86 fistulotomias, identificaram que a média deste procedimento por paciente foi de $1,2{ }^{19}$. Van Tets et al., num estudo que avaliou 312 pacientes com fístula anal, perceberam que 214 deles já haviam sido submetidos à fistulotomias anais prévias (68,58\% dos casos) ${ }^{20}$.

Outro critério avaliado foi a origem dos pacientes. No presente estudo, os pacientes operados formaram um grupo misto, procedentes tanto do sistema público quanto do sistema privado de saúde. Os grupos apresentaram distribuição semelhante nesta casuística: sistema privado $(n=214 / 49,8 \%)$ e sistema público $(n=216 / 50,2 \%)$. Este item poderia representar outro viés no trabalho, uma vez que, por se tratar de um hospital universitário, teoricamente, os pacientes do sistema público são operados pelos médicos residentes, com supervisão dos preceptores, e, os pacientes do sistema privado são operados pelos preceptores, com o auxílio dos residentes. Apesar deste fato, não houve diferença significativa se comparado o número de complicações entre os 2 grupos (52 pacientes do sistema privado e 50 do sistema público/ $p=0,863$ ).

Outro possível viés deste estudo reside no fato de que todos os pacientes não foram operados pelo mesmo cirurgião. Houve diferentes cirurgiões e equipes, componentes do serviço, que operaram os pacientes. Entretanto, houve padronização técnica em todos os procedimentos, de acordo com as condutas uniformes do serviço utilizadas ao longo do estudo.

Comparando-se a técnica anestésica empregada para a realização das operações anorretais, foi utilizada a anestesia combinada com bloqueio perianal associado à anestesia endovenosa com propofol em 374 procedimentos $(82,56 \%)$, e bloqueios espinhais nos demais $(n=79 / 17,44 \%)$. Esta diferença se dá devido a escolha da técnica de anestesia combinada como a primeira opção no serviço, devido a realização prévia de outro estudo que definiu o protocolo de rotina do serviço ${ }^{4}$. 
Análise das Complicações Tardias em Operações Anorretais

Experiência de um Serviço de Referência em Coloproctologia Juliana Stradiotto Steckert e Cols.
Vol. 30
A realização de operações em regime ambulatorial, não é algo novo na literatura ${ }^{3}$. A alta prevalência das doenças anorretais e o impacto econômico das suas variadas formas de tratamento envolvidas têm estimulado o interesse pelo manejo ambulatorial destas afecções ${ }^{3,4,5,10,21}$. As longas filas de espera de vagas para realização de operações em regime de internação hospitalar evidenciam a dimensão do problema. A cirurgia ambulatorial proctológica aparece na tentativa de se diminuir custos hospitalares e da liberação de leitos para cirurgias mais complexas ${ }^{3,4}$. A anestesia combinada parece contribuir neste cenário, como uma técnica que visa boas condições operatórias, agilidade no ambiente cirúrgico, poucos efeitos colaterais, rápida recuperação com possibilidade de alta precoce e baixo custo ${ }^{4}$.

Kotze et al. demonstraram que as operações anorretais realizadas sob anestesia combinada (propofol endovenoso associado ao bloqueio perianal local) apresentaram menor tempo de ocupação de sala cirúrgica quando comparadas às realizadas com bloqueios regionais. Isto gera um benefício direto para o hospital, bem como para as equipes médicas cirúrgicas e de anestesiologia, pela maior disponibilidade de tempo para se realizar outras operações ${ }^{4}$.

Apesar das vantagens, sejam elas sociais e/ou econômicas, ainda há relutância por parte de alguns cirurgiões e anestesiologistas para a indicação de procedimentos cirúrgicos anorretais em regime ambulatorial. As principais restrições ocorrem por receio das complicações pós-operatórias precoces, controle inadequado da dor e baixa remuneração ${ }^{3,10}$. Algumas vezes, a resistência vem do próprio paciente ${ }^{10}$. Porém, estudos brasileiros já evidenciaram as vantagens desta técnica ${ }^{3,5,10,11,21}$, o que justifica a opção do serviço que realizou este estudo em realizá-la como rotina. Salienta-se que não há trabalhos na literatura que mostrem diferenças nas taxas de complicações cirúrgicas tardias entre procedimentos anorretais realizados em regime ambulatorial e em pacientes com internação hospitalar. Há diferenças no que tange a complicações precoces. O regime de internação e a técnica de anestesia não parecem influir em maiores ou menores complicações tardias após estes procedimentos.

Para se analisar as operações realizadas, estas foram divididas em 12 grupos: hemorroidectomias, fistulotomias anais (neste grupo se encontram as fistulotomias em 1 ou 2 tempos), fissurectomias anais (associadas a esfincterotomia lateral interna ou não), cauterização de condilomas, drenagem de abscesso perianal,anopexia mecânica, biópsias anais, esfincteroplastias, anuplastias, e cirurgias mistas associadas (hemorróidas com fissuras e com fístulas anais, e fissuras associadas com fístulas anais). Apesar do número pequeno de procedimentos mistos $(\mathrm{n}=18 / 4 \%)$, eles podem propiciar um viés no estudo, pois fica difícil analisá-los quanto às complicações associadas. Procedimentos mistos teriam uma tendência natural a maiores taxas de complicações.

A operação mais freqüentemente realizada foi a hemorroidectomia: 228 procedimentos isolados $(50,3 \%)$ e 15 associados a outros procedimentos $(3,3 \%)$. Estes dados refletem que a doença anorretal mais prevalente na casuística foi a doença hemorroidária, assim como observado por outros serviços $2,3,5,6,10,11,12,13,15$, A hemorroidectomia foi a operação mais realizada, representando cerca de 40\%$80 \%$ dos procedimentos, na maioria dos estudos revisados ${ }^{2,3,5,10}$. A variação da proporção dos procedimentos existe, pois em alguns estudos analisados, considerou-se como cirurgias anorretais as exéreses de cisto pilonidal, retiradas de corpo estranho do reto, cerclagens anais e ressecções de hidradenite supurativa 3, 5, 10. Estas operações não foram computadas nesta casuística.

As fistulotomias somadas as fissurectomias (em procedimentos únicos ou asssociados) representaram $34,7 \%$ dos casos. Números semelhantes foram encontrados na literatura nacional ${ }^{2,3,5}$. A cauterização de condilomas representou 5,3\% dos casos realizados. A média coincide com a demonstrada na literatura ${ }^{3,10}$. Percebe-se, portanto, uma distribuição em incidência dos tipos de operações muito semelhante do presente estudo com a literatura nacional e internacional.

Para a avaliação ambulatorial pós-operatória, o serviço determina seguimento com consultas agendadas no $15^{\circ}$ e $45^{\circ}$ dias do pós-operatório, salvo alguma necessidade especial a ser avaliada especificamente. $\mathrm{O}$ marco dos 45 dias (7 semanas) reflete o período médio de cicatrização das feridas anais, definido na literatura como entre 6 e 8 semanas $2,7,8,10,13,15,22,23$.

O tempo médio de seguimento foi de 164,7 dias (cerca de 5,4 meses), com tempo máximo de 849 dias (2,3 anos). O tempo de seguimento foi maior do que a média encontrada na literatura ${ }^{15,22,23}$. Uma justificativa para este fato é a de que alguns dos pacientes ope- 
Análise das Complicações Tardias em Operações Anorretais

Experiência de um Serviço de Referência em Coloproctologia Juliana Stradiotto Steckert e Cols.
Vol. 30 rados são portadores de outras entidades coloproctológicas que necessitam manter um acompanhamento ambulatorial mais duradouro, como constipação intestinal e pólipos colorretais.

$\mathrm{Na}$ avaliação das 453 operações, evidenciouse 102 casos de complicações tardias $(22,52 \%)$ dos casos. Somente 2 trabalhos nacionais avaliaram complicações tardias nas operações anorretais. Entretanto, ambos utilizaram critérios diferentes dos apresentados nesta casuística para avaliação. O trabalho de Sobrado et al., que avaliou 503 pacientes, num período de 8 anos, demonstrou taxa de $4,8 \%$ de complicações tardias, mas não deixa claro na sua metodologia como foi o seguimento dos pacientes, nem os critérios utilizados para se determinar uma complicação ${ }^{3}$. A retrospectiva histórica do HU/UFS, realizada por Prudente et al. , avaliou 455 operações, num período de 3 anos $^{2}$. Citase, nos resultados, que houve incidência de $10,7 \%$ de fissuras anais residuais, 3,5\% de incontinência fecal discreta e estenose anal em $1,8 \%$, somando-se $16 \%$ de complicações tardias ${ }^{2}$. Entretanto, não fíca claro no estudo se houve outras complicações no período.

Para se analisar as complicações tardias pósoperatórias, de maneira mais didática, uma separação por tipo de complicação foi realizada, para mais fácil compreensão.

As fissuras anais residuais foram, isoladamente, as complicações tardias associadas às operações anorretais mais freqüentemente encontradas. Totalizaram 55 casos (54\% das complicações). Destas, $85,5 \%$ cicatrizaram com o tratamento clínico, que consiste em cuidados locais (banhos de assento com água morna e a não-utilização do papel higiênico), dieta rica em fibras, aumento da ingestão hídrica, e uso de pomadas cicatrizantes à base de policresuleno ou esteróides 1,6,7,24. Granet, em 1967, descrevia o quanto as fissuras anais residuais o deixavam descontente, e o faziam submeter grande parte dos seus pacientes a novos procedimentos cirúrgicos ${ }^{9}$. Neste trabalho, salienta-se ainda que as confecções de grandes feridas, com pontes de pele estreitas, resultariam numa ferida inelástica, favorecendo o aparecimento das fissuras residuais. Além disso, o trauma repetido das evacuações, lacerando-se ainda mais o tecido inelástico, poderia contribuir para este aparecimento. Para se evitar o desenvolvimento das fissuras residuais, portanto, segundo este autor, deve-se apresentar um meticuloso cuidado pós-operatório. No estudo, suas fissuras anais residuais raramente responderam ao tratamento con- servador ${ }^{9}$. A experiência desta casuística, portanto, é diferente da encontrada neste importante estudo referenciado.

A dificuldade na avaliação destes trabalhos já começa na definição da fissura anal residual. Não se sabe ao certo diferenciar ferida ainda em processo de cicatrização da fissura residual ${ }^{9}$. Talvez, no período avaliado, próximo a sete semanas de pós-operatório, ainda não tivesse ocorrido completa cicatrização das feridas operatórias, e a equipe tenha denominado este fato como uma fissura anal residual. Este viés justificaria o elevado número de fissuras residuais encontradas, bem como o enorme sucesso de cicatrização apresentado pelo tratamento clínico.

As incidências de fissuras anais residuais encontradas na literatura variam muito, pela dificuldade de se padronizar a definição deste quadro. Prudente et al. encontraram 10,7\% de fissuras residuais ${ }^{2}$; Cruz et al encontraram 2,7\% ${ }^{13}$, Ganchrow et al 2,65\% ${ }^{15} \mathrm{e}$ Nahas et al 2,5\% ${ }^{11}$, avaliando-se somente hemorroidectomias . No presente estudo, a principal operação que resultou na fissura anal residual foi a hemorroidectomia ( $p<0,0001)$.

A incontinência anal leve, caracterizada por perda do controle na eliminação de gases e secreção fecal principalmente após as evacuações (escape), foi demonstrada como a segunda mais frequiente complicação pós-operatória, ocorrendo em 13,7\% dos casos. A avaliação subjetiva de dados de incontinência, sem escores para a real avaliação por parte dos pacientes, ainda de forma retrospectiva, com dados escritos ou omitidos nos prontuários, faz deste achado um valor duvidoso nesta análise. O único trabalho que avaliou continência anal de maneira retrospectiva, no pós-operatório tardio nas operações anorretais, encontrou 3,5\% de incontinência para flatos ${ }^{2}$.

Desordens de continência anal são freqüentemente negligenciadas, mas são complicações comuns após operações anorretais, principalmente fistulotomias ${ }^{20,25}$. Incontinência leve após fistulotomias anais ocorrem em até $30 \%$ dos casos ${ }^{20,25}$. As fissurectomias anais, ainda mais quando associadas a esfincterotomias internas, também são responsáveis por alterações na continência. Nyam e Pemberton avaliaram 585 fissurectomias anais associadas a esfincterotomias, e encontraram $6 \%$ dos pacientes com incontinência para gases e $8 \%$ com escape fecal ${ }^{13}$. Analisando-se somente hemorroidectomias, Ganchrow avaliou 2038 procedimentos e não encontrou nenhum 
paciente com queixas de piora de continência pós-operatória ${ }^{26}$. Cruz et al. estudaram 2417 casos, e descreveram $0,2 \%$ dos pacientes com desordens da continência ${ }^{13}$. A variabilidade destes achados pode ser explicada pela metodologia da maioria dos estudos (retrospectiva), bem como pela ausência de padronização na literatura de adequado método de análise destes sintomas.

As fístulas residuais também se apresentaram como significativa complicação tardia $(11,8 \%$ dos casos) na presente casuística. A média relatada pela literatura fica bem aquém da encontrada nesta casuística, com valores entre $0,25 \%$ e $1,2 \%{ }^{14,15}$. Não se encontrou uma justificativa para este fato. A principal operação que resultou em fístula anal residual foi hemorroidectomia $(p=0,46)$, talvez por ser a mais freqüente na presente casuística.

O objetivo do tratamento cirúrgico das fístulas é a erradicação do trajeto fistuloso, pela identificação correta do orifício interno, sem se prejudicar a continência dos pacientes ${ }^{17}$. A taxa de recidiva de fístulas nesta casuística foi de $8,8 \%$. A média encontrada na literatura foi semelhante. Jórdan et al. avaliaram 279 casos de fistulotomias, e identificaram recidiva em 7,2\% dos casos ${ }^{27}$. Sobrado et al. encontraram 5,8\% de recidivas de fístulas ${ }^{3}$.

A recidiva de condilomas após cauterização cirúrgica ocorre entre $20 \%-30 \%$ dos casos ${ }^{\mathrm{s}}$. A taxa de recidiva neste trabalho foi de $6,8 \%$, mais baixa que os achados da literatura. No presente serviço optouse por adotar o tratamento proposto por Nadal et al., onde inicialmente é realizado o tratamento tópico com podofilina $25 \%$, o que proporciona a redução do tamanho dos condilomas, tornando a ressecção cirúrgica mais fácil ${ }^{28}$. Além disso, a utilização da medicação tópica prévia pode prolongar o período sem recidivas ${ }^{28}$.

A estenose anal é uma complicação pós-operatória que piora e muito a qualidade de vida dos pacientes. Felizmente, não é tão frequiente. Os valores encontrados na literatura variam entre 0 e $1,8 \% 2,3,13$, 14,15. A média encontrada nesta casuística foi de $2,9 \%$, portanto, um pouco além da referida pelos trabalhos revisados.

O termo "recidiva hemorroidária" não é muito bem definido nos trabalhos da literatura. Também pode ser denominado como mamilo hemorroidário residual, uma vez que não se tem a certeza da sua origem ${ }^{9}$. Muitos pacientes apresentam varizes hemorroidárias que manifestam sintomas pouco tempo após a confec- ção da hemorroidectomia. Nestes casos, é bem provável que o tecido hemorroidário fora totalmente removido. Portanto, o termo recorrência seria errôneo. Varicosidades que são removidas não podem recorrer. A "recorrência" consiste nas vênulas que, devido ao seu aspecto normal na ocasião da hemorroidectomia, passam despercebidas, e, ao passar dos anos, podem culminar em sintomas semelhantes aos de hemorróidas. É o que pode acontecer quando pacientes muito jovens são submetidos a hemorroidectomias ${ }^{9}$. A média de recorrência de hemorróidas encontrada nesta casuística foi de $2 \%$, concordando com as referidas pela literatura $(0 \%-7,2 \%)^{3,13,14,15}$.

Avaliando-se as complicações, percebeu-se a necessidade de tratamento cirúrgico em $37,23 \%$ dos casos, resultando numa taxa global de $8,83 \%$ de reoperações, entre os 430 pacientes desta casuística. Somente um trabalho avaliou a taxa de reoperações das operações anorretais, que foi de $1 \%$. Entretanto, não foi identificado qual procedimento realizado, nem a origem da complicação ${ }^{10}$. O tipo principal de reoperação foi fistulotomia anal $(40,1 \%$ dos casos das complicações), que compreendia pacientes com fístulas anais recidivadas e fístulas residuais. Somente 14,5\% dos pacientes com fissura residual necessitaram de reoperação. Estes achados são comparáveis aos da presente casuística.

$\mathrm{Na}$ tentativa de se procurar por fatores de risco para complicações (fatores que pudessem estar implicados num aumento das complicações tardias das operações anorretais), comparou-se a distribuição de homens e mulheres nos casos de complicações, e não houve diferença significativa $(p=0,53)$. Também se distribuíram as complicações de acordo com a faixa etária dos pacientes acometidos, e nenhum grupo se destacou $(p=0,582)$. Analisou-se a procedência dos pacientes, e não houve diferença entre o grupo de pacientes do sistema público e do privado na ocorrência de complicações $(p=0,863)$. Subdividiu-se os tipos de procedimentos cirúrgicos correspondentes a cada complicação, mas também não houve diferença entre o procedimento realizado e as complicações encontradas ( $p=$ $0,107)$. Apenas na análise isolada das fissuras anais residuais, destacou-se a hemorroidectomia como principal causa, com significância estatística. Não foram encontrados estudos na literatura que analisassem os mesmos dados para se traçar uma comparação.

Em primeira análise, as taxas de complicações tardias encontradas após operações sobre o anorreto 
Rev bras Coloproct Julho/Setembro, 2010
Análise das Complicações Tardias em Operações Anorretais: Experiência de um Serviço de Referência em Coloproctologia Juliana Stradiotto Steckert e Cols.
Vol. 30 podem ser consideradas altas na presente casuística. Estes dados foram alarmantes e serão usados para cada vez mais se procurar uma excelência na técnica cirúrgica destes procedimentos. Este é o valor de um serviço universitário, onde se procura o porquê dos fatos, com uma aplicabilidade direta no benefício dos pacientes a serem operados no futuro.

\section{CONCLUSÕES}

Após a análise dos dados da presente casuística, pode-se chegar as seguintes conclusões:

A taxa de complicações tardias decorrentes das operações anorretais realizadas no serviço universitário foi de $22,52 \%$.

Os dados epidemiológicos associados aos pacientes submetidos às operações anorretais evidenciaram grupo semelhante quanto ao sexo. A média de idade foi de 44,3 anos.
As fissuras anais residuais foram as complicações mais freqüentes, e tiveram a hemorroidectomia como sua principal causa, com significância estatística.

A taxa de reoperações no presente estudo foi de $8,83 \%$ do total de casos.

Não foram encontrados fatores de risco que justificassem o aumento nas taxas de complicações tardias em geral, nas operações anorretais. Os valores foram semelhantes quando se comparou sexo, faixa etária, procedência do paciente e o tipo de cirurgia realizada.

\section{AGRADECIMENTOS}

Agradecemos aos Doutores Luis Carlos von Bahten (Chefe do Serviço de Cirurgia Geral do Hospital Universitário Cajuru- PUCPR) e Rubens Valarini (Chefe do Serviço de Coloproctologia do Hospital Evangélico-PR) pelas sugestões dadas a este trabalho.

\begin{abstract}
Introduction: anorectal procedures consist $80 \%$ of surgical cases in colorectal surgery practice. The exact rate of long-term complications after anorectal surgery is unknown. This number is variable according to the medical centres and the type of procedures. Objective: to evaluate the long-term complications secondary to anorectal procedures, as well as the risk factors that might be associated with these complications. Method: retrospective analysis, including anorectal procedures performed between January 2007 and July 2009. The characteristics analyzed were: sex, age, type of surgery, health system, long-term complications and reoperations performed. Results: 430 patients submitted to 453 anorectal procedures were studied (54,4\% female). Hemorrhoidectomy was the most common procedure (50,3\% of all operations). The mean period of follow-up was 164,7 days and 102 long-term complications were identified, occurring in $22,52 \%$ of all procedures. Residual fissure in ano was the most frequent complication $(54 \%, n=55)$. Only 38 patients needed reoperation $(8,83 \%$ of all cases $)$. There was no statistical significance between sex, age, health system and type of surgery in relation to the complications found. Conclusions: the long-term complication rate was $22,52 \%$, with reoperations performed in $\mathbf{8 , 8 3} \%$ of all patients. There was no risk factor for long-term complications identified in this case series.
\end{abstract}

Key words: Postoperative complications, hemorrhoids, rectal fistula, fissure in ano, condylomata acuminata.

\section{REFERÊNCIAS}

1. Kotze, PG; Martins, JF; Steckert, JS. Operações Anorretais. [acesso 10 set. 2009]. Curitiba; 2009. Disponível em:<http:// www.cbc.org.br/upload//1251169200120146.pdf > .

2. Prudente, ACL; Neto, JRT; Santiago, RR; Mariano, DR; Vieira Filho, MC. Cirurgias proctológicas em 3 anos de serviço de coloproctologia: série histórica. Rev bras Coloproct. 2009; 29(1): 71-76.

3. Sobrado, CW; Nahas, CF; Habr-Gama, A. Cirurgia ambulatorial sob anestesia local em proctologia: experiência e análise do resultado de 503 operações. Rev bras Coloproct 2001; 21(4): 228-233.
4. Kotze, PG; Tambara, EM; Von Bahten, LC; Silveira, F; Wietzikoski, E. Influência da técnica de anestesia no tempo de ocupação da sala cirúrgica nas operações anorretais. Rev bras Coloproct 2008; 28(2): 227-233.

5. Nahas, SC; Sobrado, CW; Marques, CF; Imperiale, AR; HabrGama, A; Rocha, JP; Auler Júnior, JO. Orifice diseases projectexperience of the "Hospital das Clínicas" University of São Paulo Medical Center in day-hospital of anorectal disease. Rev Hosp Clín Fac Med S Paulo 1999; 54(3): 75-80.

6. Cintron, JR; Abcarian H. Benign anorrectal: hemorrhoids. In: Wolff, BG; Fleshman, JW; Beck, DE; Pemberton, JH; Wexner, SD. The ASCRS textbook of colon and rectal surgery. New York: Springer; 2007. p.156-171. 
7. Dykes, SI; Madoff, RD. Benign anorrectal: anal fissure. In: Wolff, BG; Fleshman, JW; Beck, DE; Pemberton, JH; Wexner, SD. The ASCRS textbook of colon and rectal surgery. New York: Springer; 2007. p.178-187.

8. Vasilevsky, CA; Gordon, PH. Benign anorrectal: abscess and fistula. In: Wolff, BG; Fleshman, JW; Beck, DE; Pemberton, JH; Wexner, SD. The ASCRS textbook of colon and rectal surgery. New York: Springer; 2007. p.192-210.

9. Granet, E. Hemorrhoidectomy failures: causes, prevention and management. Dis Colon Rectum 1968; 11(1): 45-48.

10. Saad-Hossne, R; Prado, RG; Bakonyi-Neto, A. Cirurgia ambulatorial em coloproctologia: análise retrospectiva de 437 casos. Arq Gastroenterol 2005; 42(3): 136-138.

11. Nahas, SC; Sobrado, CW; Araújo, SEA; Imperiale, AR; HabrGama, A; Pinotti, HW. Resultados do tratamento cirúrgico da doença hemorroidária em 475 pacientes. Rev Hosp Clín Fac Med S Paulo 1997; 52(4): 175-179.

12. Pinho, MSLP; Ferreira, LC; Vasconcelos, ECG; Filho, NAS; Reis, MC. Análise da prevalência por sexo e idade nas doenças anorretais freqüentes. Rev bras Coloproct 2002; 22(3): 158163.

13. Cruz, GMG; Santana, JL; Santana, SKAA; Ferreira, RMRS; Neves, PM; Faria, MNZ. Hemorroidectomia: estudo de 2417 pacientes submetidos à cirurgia para tratamento da doença hemorroidária. Rev bras Coloproct 2006; 26(3): 253-268.

14. McConnell, JC; Khubchandani, IT. Long-term follow-up of closed hemorrhoidectomy. Dis Colon Rectum 1983; 26(12): 797-799.

15. Ganchrow, MI; Mazier, WP; Friend, WG; Fergunson, JA. Hemorrhoidectomy revisited - a computer analysis of 2038 cases. Dis Colon Rectum 1971; 14(2): 128-133.

16. Chaves, DNB; Petroniau, A; Oliveira, HM; Capobiango, A. Avaliação pós-operatória tardia de pacientes submetidos à esfincterotomia anal interna. Rev bras Coloproct 2000; 20(3): 168-171.

17. van Koperen, PJ; Wind, J; Bemelman, WA; Bakx, R; Reitsma, JB; Slors,FM. Long-term functional outcome and risk factors recurrence after surgical treatment for low and high perianal fistulas of cryptoglandular origin. Dis Colon Rectum 2008; 51(10): 1475-1481.

18. Nyam, DCNK; Pemberton, JH. Long-term results of lateral internal sphincterotomy of chronic anal fissure with particular reference to incidence of fecal incontinence. Dis Colon Rectum 1999; 42(10): 1306-1310.
19. Davies, M; Harris, D; Lohana, P; Chandra Sckaran, TV; Morgan, AR; Beyon, J; Carr, ND. The surgical manangement of fistula-in-ano in a specialist colorrectal unit. Int J Colorectal Dis 2008; 23(9): 833-838.

20. van Tets, WF; Kuijpers, HC. Continence disordes after anal fistulotomy. Dis Colon Rectum 1994; 37(12):1194-1197.

21. Kotze, PG; Martins, JF; Steckert, JS; Rocha, JG; Sartor, MC; Miranda, EF. Anopexia mecânica com anestesia combinadaexperiência inicial. Rev Méd Res 2009; 11(2): 54-61.

22. MacRae, HM; McLeod, RS. Comparison of hemorrhoidal treatment modalities. Dis Colon Rectum 1995; 38(7): 687694.

23. Cruz, GMG; Santana, JL; Santana, SKAA; Ferreira, RMRS; Neves, PM; Faria, MNZ. Hemorroidectomia: estudo de 2417 pacientes submetidos à cirurgia para tratamento da doença hemorroidária. Rev bras Coloproct 2006; 26(3): 253-268.

24. Sousa, MM. Tratamento clínico da fissura anal crônica. Estudo comparativo entre diltiazem $1 \%$ e betanecol $0,1 \%$. [acesso 05 set. 2009]. Curitiba; 2009. Disponível em: <http:// www.teses.usp.br/teses/disponiveis/5/5154/tde-24112008$113247 />$.

25. Campos, FGCM; Araújo, SEA; Habr-Gama, A. Etiologia e tratamento cirúrgico das fissuras anais. Revisão sobre aspectos históricos, técnicos e resultados. Rev bras Coloproct 2001; 21(4): 239-245.

26. Moesgaard, F; Nielsen, ML; Hansen, JB; Knudsen,JT. Highfiber diet reduces bleeding and pain in patients with hemorrhoids: a double-bind trial of Vi-Siblin. Dis Colon Rectum 1982; 25(5): 454-456.

27. Jordán, J; Roig, JV; García-Armengol, J; García-Granero, E; Solana, A; Lliedó, S. Risk factors for recurrence and incontinence after anal fistula surgery. Colorectal Disease. No prelo 2009.

28. Nadal, SR; Manzione, CR; Horta, SHC; Calore, EE. Sistematização do atendimento dos portadores de infecção perianal pelo papilomavírus humano (HPV). Rev bras Coloproct 2004; 24(4): 322-328.

Endereço de correspondência:

JULIANA STRADIOTTO STECKERT

Av. Buriti, 620 ap 806B CEP 88034-500

Parque São Jorge - Florianópolis/SC

Fone: (48) 32348095

E-mail: justeckert@hotmail.com 